\title{
Effect of single-incision plus one port laparoscopic surgery assisted with enhanced recovery after surgery on colorectal cancer: study protocol for a single-arm trial
}

\author{
Xuehua Zhang ${ }^{1 \#}$, Gaohua Li ${ }^{1 \#}$, Xiaojing Li ${ }^{2 \#}$, Zhenye Liang ${ }^{1}$, Xiaoliang Lan ${ }^{1}$, Tingyu Mou ${ }^{1}$, \\ Zhenzhao $\mathrm{Xu}^{1}$, Jie $\mathrm{Fu}^{1}$, Mingyi $\mathrm{Wu}^{1}$, Guoxin $\mathrm{Li}^{1}$, Yanan Wang ${ }^{1}$ \\ ${ }^{1}$ Department of General Surgery, Nanfang Hospital, Southern Medical University, Guangzhou, China; ${ }^{2}$ The First School of Clinical Medicine, \\ Nanfang Hospital, Southern Medical University, Guangzhou, China \\ Contributions: (I) Conception and design: G Li, Y Wang; (II) Administrative support: G Li, Y Wang; (III) Provision of study materials or patients: \\ X Zhang, G Li, X Li; (IV) Collection and assembly of data: J Fu, M Wu; (V) Data analysis and interpretation: Z Liang, Z Xu; (VI) Manuscript \\ writing: All authors; (VII) Final approval of manuscript: All authors. \\ "These authors are contributed equally to this work. \\ Correspondence to: Yanan Wang, MD; Guoxin Li, MD. Professor of Medicine, Department of General Surgery, Nanfang Hospital, Southern Medical \\ University, Guangzhou, China. Email: wyn8116@163.com; gzliguoxin@163.com.
}

Background: Studies have proved that the enhanced recovery after surgery (ERAS) protocol can significantly improve the recovery course of patients during the perioperative period. The application of minimally invasive surgery is a critical component of ERAS protocol. Single-incision plus one port laparoscopic surgery (SILS plus one) could achieve further minimally invasive surgical results than conventional laparoscopic surgery (CLS). The objective of this trial is to evaluate the safety and feasibility of SILS plus one with ERAS protocol in colorectal cancer.

Methods: This is a prospective, single-center, open-label, single-arm trial. A total of 120 eligible patients with colorectal cancer will receive SILS plus one followed by the ERAS management during the perioperative period. The primary endpoint is postoperative hospital stay. The secondary endpoints include rehabilitative rate of the fourth postoperative day, postoperative medical cost, postoperative pain score, postoperative recovery indexes, inflammatory immune response indexes, compliance with ERAS measures, 6 min postoperative walking test (6MWT), hospital readmissions, and early postoperative complications.

Discussion: This trial will be the first to evaluate the short-term outcomes of SILS plus one assisted with ERAS protocol for patients with colorectal cancer and will provide valuable clinical evidence on the benefit of the combination of these two techniques, hopefully, to provide patients with more safe, economic, feasible, and rapid surgery and perioperative strategies.

Trial Registration: Clinical Trial Registry, NCT0426829. Registered February 15, 2020 (https:// clinicaltrials.gov/ct2/show/NCT04268290).

Keywords: Colorectal cancer; laparoscopic surgery; single-incision plus one port laparoscopic surgery (SILS plus one); enhanced recovery after surgery (ERAS); study protocol

Submitted Jul 20, 2021. Accepted for publication Nov 05, 2021.

doi: $10.21037 /$ tcr-21-1361

View this article at: https://dx.doi.org/10.21037/tcr-21-1361 


\section{Introduction}

Colorectal cancer is the third most common cancers worldwide, with 2 million new cases and approximately 600,000 deaths every year (1). Radical surgery has remained the primary treatment strategy for patients with colorectal cancer. In recent years, the development of laparoscopy and implementation of enhanced recovery after surgery (ERAS) protocol has established a more minimally invasive treatment for colorectal cancer.

ERAS, also known as fast track surgery, was first proposed by Henrik Kehlet in the 1990s (2). ERAS protocol is an evidence-based perioperative management strategy, mainly including preoperative education and consultation, no mechanical bowel preparation, restrictive fluid infusion, postoperative analgesia, early feeding, and early ambulation. With its widespread application into various disciplines, increasing studies have shown positive evidence of the benefits of ERAS, including better postoperative recovery, earlier hospital discharge, less medical costs, and lower complication rate (3-7). Besides, ERAS protocol for patients with colorectal cancer has achieved significant results of faster postoperative recovery (8). Europe (9) and Japan (10) have issued guidelines and consensus for the ERAS on colorectal cancer surgery in 2013 and 2015, respectively. These years, Chinese guidelines have also recommended ERAS as perioperative management for colorectal cancer surgery, which further affirmed the role of ERAS in colorectal surgery and standardized ERAS protocol based on medical evidence.

In terms of ERAS concept, surgical approach plays an integral part in the patient's recovery process. Over the last two decades, laparoscopic surgery for colorectal cancer has been accepted as a preferred alternative to conventional open surgery $(11,12)$. Recently, attempts have been made on single-incision laparoscopic surgery (SILS) to further reduce abdominal incisions. The SILS concept refers to the application of a single multichannel port site to perform the intraperitoneal procedure during colorectal surgery. However, due to the technical difficulty and relatively long learning curve of conducting SILS, only four prospective clinical studies concerning SILS for colorectal cancer have currently published (13-16). Accordingly, adding an additional port to SILS, known as single-incision plus one port laparoscopic surgery (SILS plus one), has been gaining more and more attention because of its essential role in bridging the gap between conventional laparoscopic surgery (CLS) and SILS. However, there is still no prospective study assessing the feasibility and short-term safety for SILS plus one assisted with ERAS for colorectal cancer.

In summary, the objective of this single-arm trial aims to evaluate the clinical benefit of SILS plus one assisted with ERAS for colorectal cancer. And hopefully, this trial can provide a more safe, economic, effective, and rapid perioperative strategy to patients with colorectal cancer. We present the following article in accordance with the SPIRIT reporting checklist (available at https://dx.doi.org/10.21037/ tcr-21-1361).

\section{Methods}

\section{Study design}

This is a prospective, single-center, open-label, singlearm trial. A total of 120 eligible patients with colorectal cancer will be recruited and will receive SILS plus one and ERAS protocol during the perioperative period at Nanfang Hospital of Southern Medical University, Guangzhou, China. The study protocol was approved by the Medical Ethics Committee of Nanfang Hospital (reference number: NFEC-2019-156). The study will be conducted in accordance with the Declaration of Helsinki (as revised in 2013). The Medical Ethics Committee of Nanfang Hospital will be informed of significant protocol amendments. Written informed consent will be obtained from all individual participants for publication of this study. This trial has been registered at Clinical-Trials.gov under the registration number NCT0426829 on February 15, 2020.

\section{Participant selection}

Patients diagnosed as radical colorectal cancer by abdominal computed tomography and colonoscopy according to the 7th Edition of the AJCC Cancer Staging Manual and feasible for SILS plus one, will be further screened for inclusion by a designated investigator (17). The inclusion, exclusion, and withdraw criteria are shown in detail in Table 1, and the flow chart for this trial is shown in Figure 1.

\section{Operative approach}

Surgery will be performed by a surgeon with the experience of conducting over 300 successful transumbilical SILS plus one colorectal resections.

After the induction of general anesthesia, patients 
Table 1 Inclusion, exclusion, and withdrawal criteria

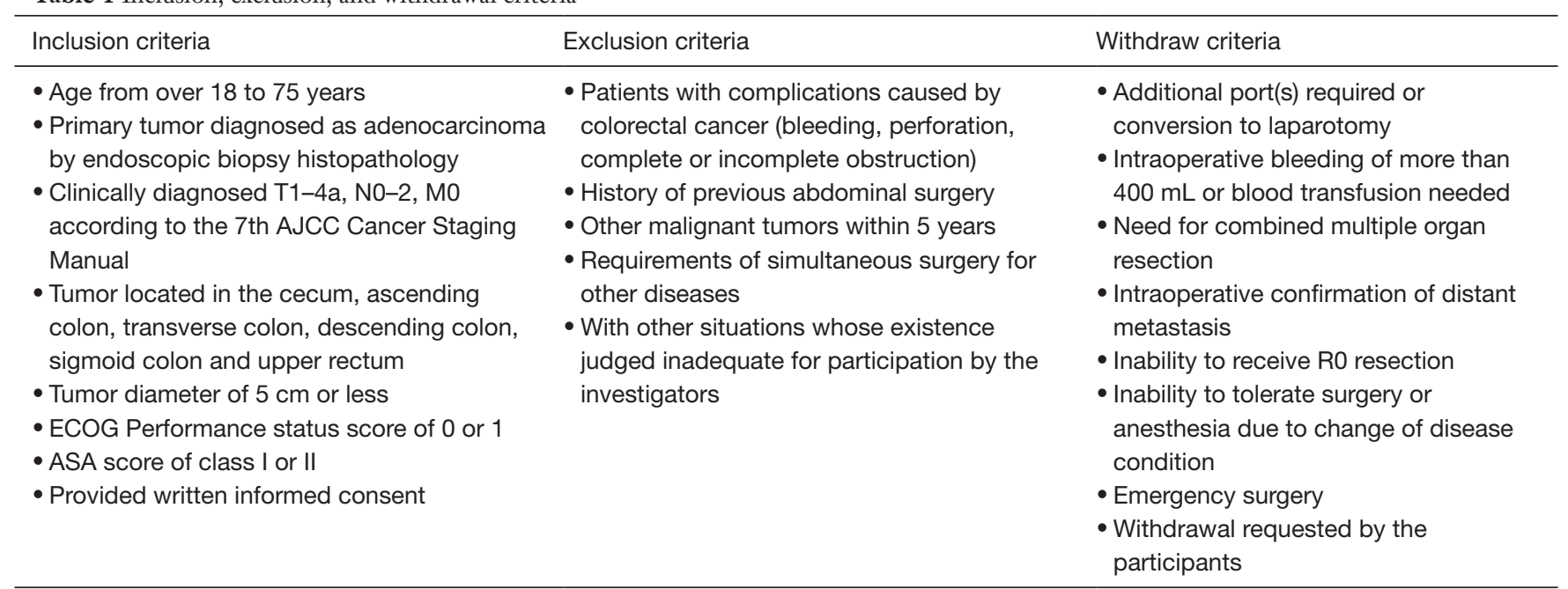

AJCC, American Joint Committee on Cancer; ECOG, Eastern Cooperative Oncology Group; ASA, American Society of Anesthesiology.

with right colon cancer will be placed in a supine split leg position, and those with left colon, sigmoid colon or upper rectal cancer will be placed in a lithotomy position on the table. An initial 3-5-cm vertical periumbilical incision will be made. Then an independently developed multiport device (Surgaid Medical; Xiamen, China) will be inserted via the umbilical incision (Figure 2). An additional 5-mm trocar will be placed in the left lower quadrant and serve as the surgeon's dominant operating channel when performing right hemicolectomy, while a $12-\mathrm{mm}$ trocar will be added in the right lower quadrant when conducting the resection of left-sided, sigmoid colon or upper rectal tumors (Figure 3). The surgeon's dominant operating channel can be adjusted slightly in accordance with the position of the tumor. Tumor location and clinical stage assessed before surgery will determine the extent of surgical resection and lymph node dissection.

The intraperitoneal procedures, including mobilization and lymph node dissection, will be the same as those of CLS (18). After mobilization, the specimen will be retrieved through the periumbilical incision where the multiport device was placed. Adding ports or converting to open surgery will be encouraged at the surgeon's discretion if technical difficulties, patient's safety, or unexpected conditions that require conversion.

\section{Perioperative management of ERAS protocol}

\section{Before surgery}

After evaluation for eligibility, patients will be asked to sign an informed consent to participate in the trial and undergo preoperative assessments. Preoperative education and consultation will provide patients with education about perioperative dietary guidance, perioperative respiratory training, and the guidance of atomization and postoperative rehabilitation. Patients with 3 or more nutritional risk screening 2002 scores will be given enteral nutrition (EN) for 5 to 7 days (500 to $1,000 \mathrm{~mL} / \mathrm{d}$, standard $\mathrm{EN}$ fluids). However, parenteral nutrition will be applied if EN fails. For patients undergoing right hemicolectomy, general diets will be maintained until $24 \mathrm{~h}$ before surgery. For those undergoing left hemicolectomy, sigmoid colectomy or rectectomy, soft diets will be conducted from three days to $24 \mathrm{~h}$ before surgery. Then all the patients will change to EN preparations of standard concentration until $2 \mathrm{~h}$ before surgery. Eventually, $200 \mathrm{~mL}$ of $10 \%$ glucose solution will be given orally before surgery (water drinking as an alternative for diabetics). For bowel preparation, patients undergoing right hemicolectomy will be given oral lactulose solution the day before surgery ( 3 times/day, $30 \mathrm{~mL}$ per time). Instead, oral lactulose solution will be used to promote bowel movement for those undergoing left hemicolectomy, sigmoid colectomy or rectectomy three days before the operation. Bowel preparation should comply with the requirement of colonoscopy if colonoscopy is needed preoperatively or intraoperatively. However, mechanical enema will not be routinely conducted. Also, preoperative nasogastric tubes will not be used routinely. If bloating affects the operation, a nasogastric tube can be temporarily indwelled but should be removed before the reversal of 


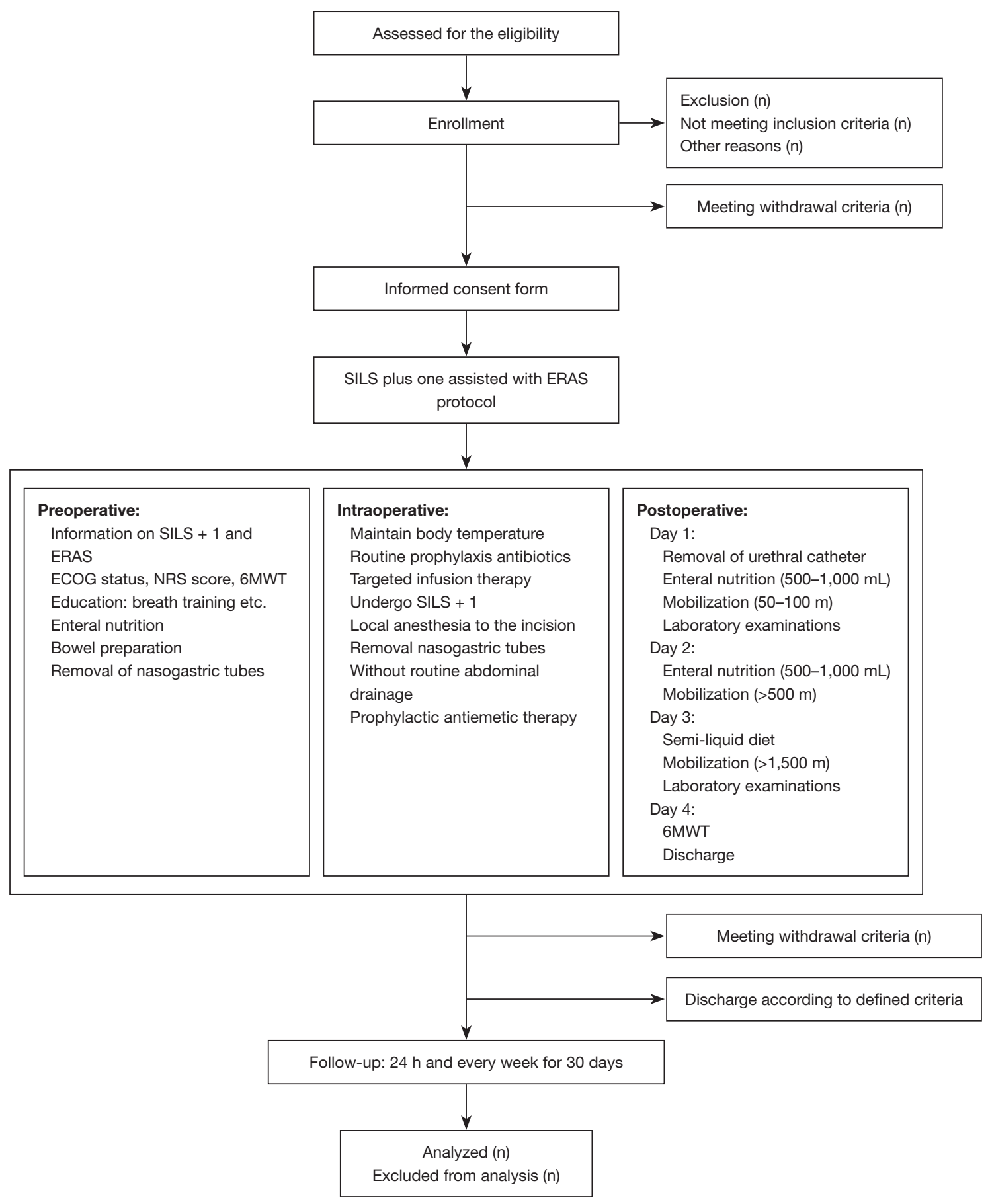

Figure 1 Flowchart. ERAS, enhanced recovery after surgery; ECOG, Eastern Cooperative Oncology Group; 6MWT, 6 min postoperative walking test.

anesthesia.

\section{During surgery}

All patients will undergo SILS plus one. When positioning the patient, theatre nurses will confirm that patients wear well-fitted compression stockings with intermittent pneumatic compression. After the induction of anesthesia, patients will adopt an indwelling urinary catheter. The 

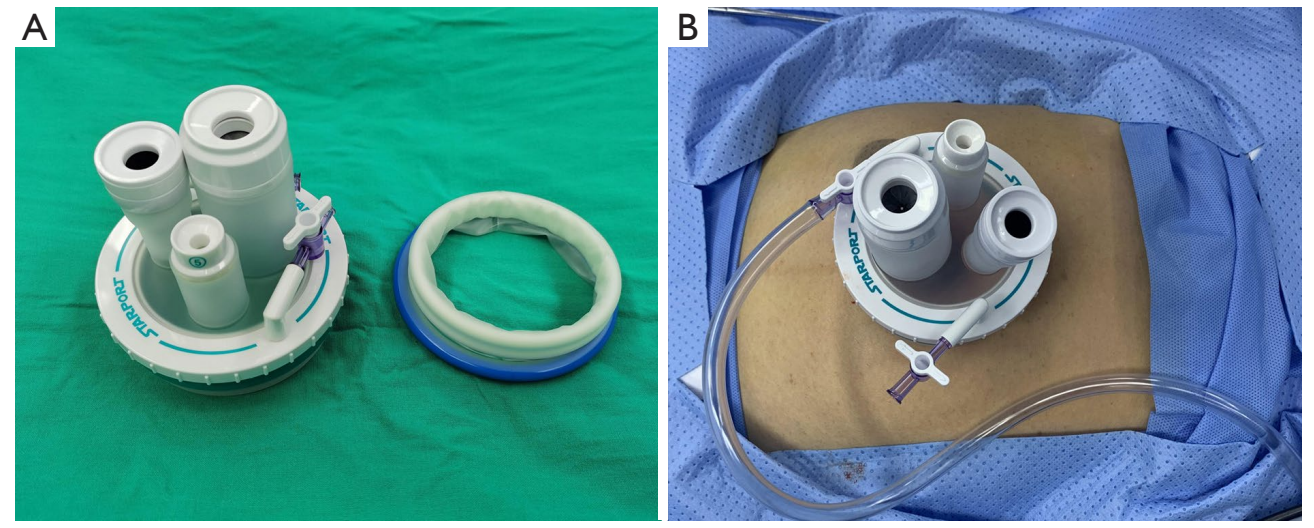

Figure 2 Single-incision multichannel device.
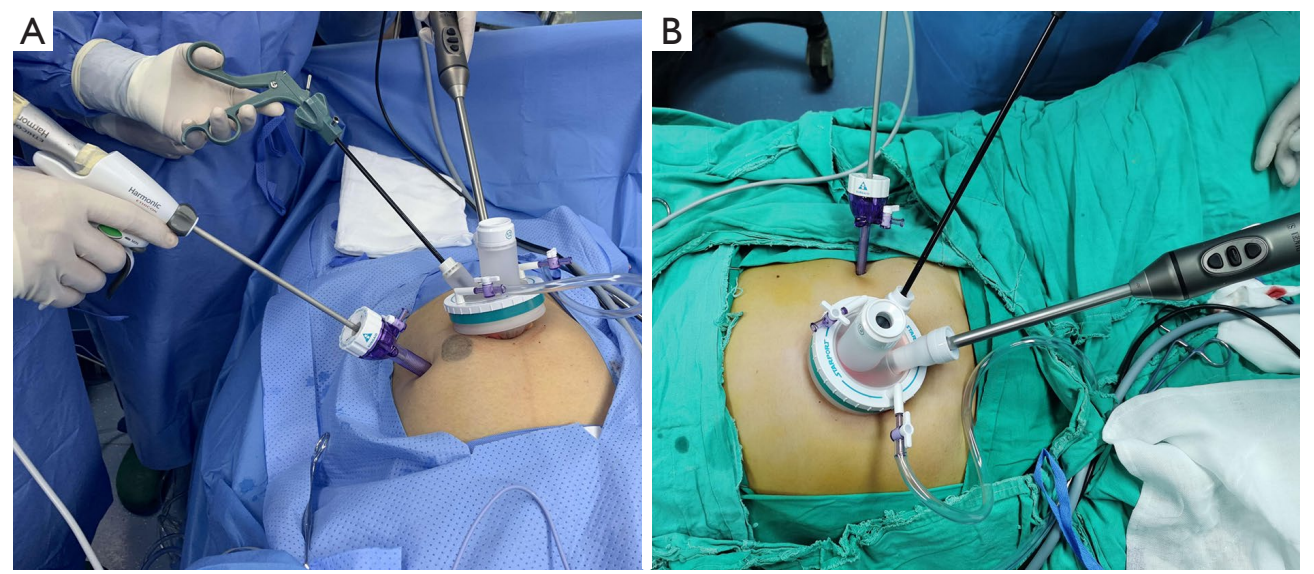

Figure 3 Trocars and instrument positions for SILS plus one. (A) Trocars and instrument position for left hemicolectomy, sigmoid colectomy or rectectomy. (B) Trocars and instrument positions for right hemicolectomy. SILS plus one, single incision plus one port laparoscopic surgery.

body temperature of patients will be maintained between 36.5 and $37.5^{\circ} \mathrm{C}$ (measured every $30 \mathrm{~min}$ ) by using forcedair heating blankets and keeping the operation room temperature between 22 and $24^{\circ} \mathrm{C}$. Besides, intravenous fluids will be heated to $37-38^{\circ} \mathrm{C}$ with an infusion warmer before administration. Prophylactic antibiotics (secondgeneration cephalosporin) will be applied intravenously 30 to $60 \mathrm{~min}$ before surgery. If the operation takes more than $3 \mathrm{~h}$ or two half-lives of the antibiotics used preoperatively, an additional single dose will be administered intraoperatively. Analgesic drugs and proton pump inhibitors will be injected intravenously before anesthetic induction or skin incision. Intravenous anesthesia will be recommended with short-acting anesthetic inducers, opioids, and muscle relaxants. Targeted infusion therapy will be implemented for restrictive infusion. Intravenous rehydration with balanced electrolyte solutions will be adopted to supply physiological requirements (no more than $1,500 \mathrm{~mL}$ ). Vasopressin medication will be the priority to maintain blood pressure during the surgery. An abdominal drainage tube will not be placed if the operation is successful without any risks of anastomotic leakage.

\section{After surgery}

After returning to the ward, patients could drink a small amount of water for several times based on their tolerance to it and will then receive $200 \mathrm{~mL} \mathrm{EN}$ of the half standard concentration. Prophylactic analgesia and antiemetic will be administered within three days postoperatively and will be maintained until discharge if necessary. The urethral 
catheter will be removed on postoperative day 1 (POD1). Patients will be given 500 to $1,000 \mathrm{~mL}$ of EN preparations of the half standard concentration on POD1 and turn to the ones of standard concentration on POD2. Soft diet will be adopted with unlimited times on POD3. Ambulation will be encouraged after surgery. Patients will be asked to perform bedside activities for 2 to 4 times, and have a 50-100-meter walk outside the ward with walking aids or mobile infusion frame on POD1. Increasing intensity of ambulation will be encouraged after POD1, and the goal of total walking distance is expected to exceed 500 and $1,500 \mathrm{~m}$ on POD2 and POD3, respectively. The postoperative inflammatory immune index will be reexamined on POD1 and POD3. On POD4, 6 min postoperative walking test (6MWT) will be adopted, and postoperative recovery index will be recorded to assess the overall conditions of patients. Patients will be discharged with their consent when meeting the criteria.

\section{Outcome measures}

\section{Primary endpoints}

The primary endpoint is postoperative hospital stay. Postoperative hospital stay is the number of days from surgery to discharge. The discharge criteria is measured by the following items: (I) patients could tolerate soft diet postoperatively and could intake physiological need for calories orally (more than $20 \mathrm{kcal} / \mathrm{kg}$ each day); (II) no intravenous infusion is required; (III) satisfying pain control [visual analogue scale (VAS) score no more than 3 points] is achieved; (IV) patients are able to walk a predefined distance, go to the bathroom or walk outside the room independently; (V) no complication is observed.

\section{Secondary endpoints}

Secondary endpoints include rehabilitative rate of the fourth postoperative day (rehabilitative rate day 4), postoperative medical cost, postoperative pain score, postoperative recovery index, postoperative inflammatory immune response indexes, compliance with ERAS protocol, 6MWT, hospital readmission, and early postoperative complications. The rehabilitation criteria of rehabilitative rate day 4 are the same as those of discharge criteria. The medical cost will be measured from the day of surgery to discharge. Postoperative pain will be assessed using the VAS daily after the operation. The first time to early mobilization (h), flatus (h), liquid diet (h), soft diet (h), and removal of the peritoneal drainage tubes (h) will be applied to assess the postoperative recovery. Compliance with ERAS protocol is defined as whether the patients complete every ERAS measure or not. We will define it as "yes" or "no". The laboratory tests such as white blood cell count, lymphocyte count, C-reactive protein (CRP), and interleukin-6 (IL-6) will be performed on POD1 and POD3 to evaluate the postoperative inflammatory response. The results of $6 \mathrm{MWT}$ on POD4 or the day of discharge and during follow-up will be recorded. Study site, subject preparation, trial procedures and medical monitoring of $6 \mathrm{MWT}$ will comply with those recommended by the guideline issued by the American Thoracic Society in 2002 (19). Hospital readmission is defined as the number of patients readmitted within 30 days, except for those readmitted for chemotherapy. Early postoperative complications refer to complications graded according to Clavien-Dindo within 30 days (20). The detailed timeline of measuring each outcome is presented in Table 2.

\section{Follow-up}

Patients will be followed up by our follow-up team $24 \mathrm{~h}$ after discharge and every week for 30 days to assess and record the postoperative recovery and complications of participants.

\section{Data collection and management}

The surgeons involved in this trial will record the details of the surgical procedure, such as the surgical approach, the location of the tumor, the number of lymph nodes dissected, and the distal and proximal resection margin distance. From POD1, the clinical observation data (e.g., first flatus time, time to the recovery of bowel sound, time to liquid diet and soft diet, time to the removal of the abdominal drainage tube, and time to the occurrence of complications) will be documented daily by qualified and trained nurses to evaluate postoperative recovery. Clinicians will be responsible for the management of patients but not involved in the collection of clinical observation data.

A specific research nurse will record the raw data in the written form case report form (CRF) first and save it electronically afterwards. Researchers will ensure that the anonymity of subjects is maintained and that unauthorized parties are prevented from the data of the participants. Inspectors will verify and cross-check the data of CRF following the items of Good Clinical Practice to ensure the accuracy and reliability of data collection. Also, CRF data will be reviewed continuously to ensure its clinical and 
Table 2 Assessments and timepoints

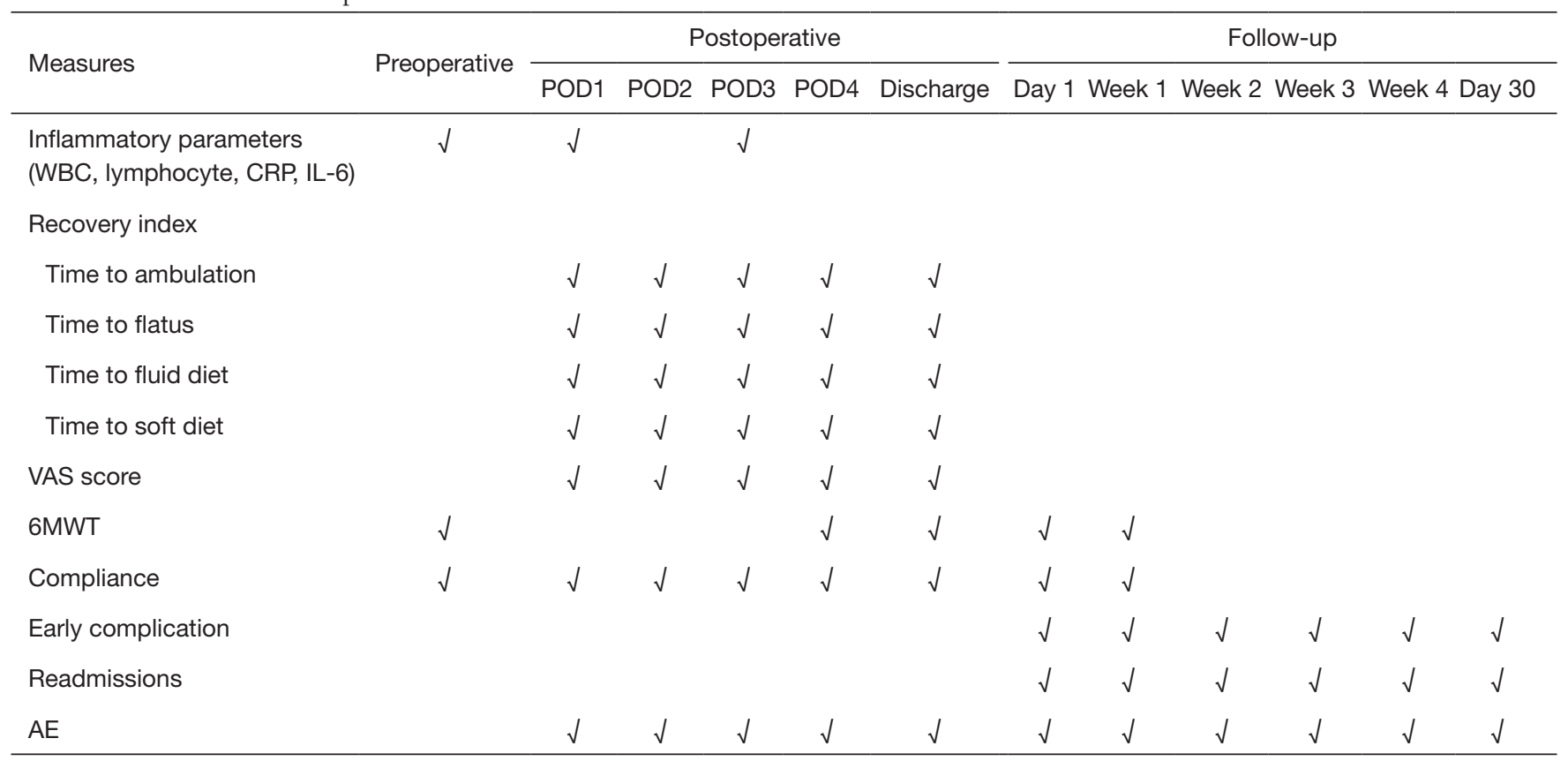

POD, postoperative of the day; WBC, white blood cell; CRP, C-reactive protein; IL-6, interleukin-6; VAS, visual analogue scale; 6MWT, 6 min postoperative walking test; $\mathrm{AE}$, adverse event.

scientifical rationality.

\section{Safety}

An adverse event (AE) is defined as any unfavorable and unintended sign (including an abnormal laboratory finding), symptom or disease occurring during the trial, which will be evaluated for severity and causality. Severity will be graded according to the CTCAE (21) and TASGS (22). Causality will be graded as none, unlikely, possibly, probably/likely or certainly related to the intervention.

Any $\mathrm{AE}$ that meets one of the following criteria is defined as a serious adverse event (SAE):

(I) Life-threatening, referring to the condition that subjects are in immediate danger of death when AEs occur rather than the condition that subjects will be in danger of death if AEs progress.

(II) Lethal. If the outcome of an event is "death" then it will be reported and recorded as SAE.

(III) Resulting in hospitalization or prolonged hospitalization, the reason leads to this condition should be explicit.

(IV) Resulting in permanent or serious disability or dysfunction.

(V) Causing malformation if giving birth to a child.
(VI) Other significant medical events.

If any $\mathrm{AE}$ or SAE occurs, the researchers will fill in the AE report form and CFDA (China Food and Drug Administration) SAE report form and report it to the chief investigator $(\mathrm{CI})$ within $24 \mathrm{~h}$.

\section{Statistical analyses}

\section{Sample size}

The sample size was determined for the primary endpoint: postoperative hospital stays. Based on our database, the average length of stay after CLS with conventional care for colorectal cancer is 7.0 days [standard deviation $(\mathrm{SD})=3.4$ ] and that after SILS + 1 assistant ERAS for colorectal cancer is 4.0 days $(\mathrm{SD}=1.2)$. The significance level and power are set at 0.05 and 0.8 , respectively. With a dropout rate of $10 \%$, a sample size of 120 participants was calculated using PASS V11.07 software.

\section{Statistical methods}

Continuous variables were expressed as the means and SDs or medians and interquartile ranges as appropriate. Categorical variables were summarized as the counts and percentages. Categorical variables will be analyzed by the Chi-squared test or Fisher's exact test and continuous 
variables will be analyzed by the $t$-test or Wilcoxon signedrank test. Any outcomes of the interest analysis will be performed based on per-protocol population (PPP) and standard sensitivity analysis will also be performed based on PPP. A P value of less than 0.05 will be considered to indicate statistical significance. Data analysis will be performed by using SPSS $^{\circledR}$ software package version 22.0 (SPSS Inc., Chicago, IL, USA).

\section{Discussion}

The ERAS protocol consists of multimodal perioperative management pathways designed to decrease surgical stress and enhance early postoperative recovery. A meta-analysis of randomized controlled trials (RCTs) has shown that compared with conventional perioperative care, the ERAS protocol leads to a reduction in hospitalization by more than two days and an almost $50 \%$ reduction in complication rates without increasing hospital readmissions in patients undergoing open colorectal surgery (23).

In terms of the ERAS concept, surgical trauma is the most critical factor contributing to surgical stress. Consequently, it is suggested that surgeons perform the operations with the notions of precision, minimallyinvasiveness, and damage control in order to reduce stress response to trauma. Besides, surgical trauma is a major cause of postoperative complications that affect directly the process of recovery.

It is generally accepted that minimally invasive surgery could reduce surgical trauma significantly, compared to conventional surgery. Over the last two decades, CLS has become the recommended surgical procedure for colorectal cancer, bringing various advantages, including less pain, shorter hospital stays, and better cosmetic results $(24,25)$. According to meta-analyses, CLS for colorectal cancer could yield comparable short- and long-term oncologic outcomes with open surgery (26-28). Recently, several RCTs demonstrated that the CLS optimized by ERAS protocols could result in much less trauma, less blood loss, and earlier flatus and defecation for patients with colorectal cancer (29-31). Hence, patients with colorectal cancer are more likely to benefit from the combination of the ERAS protocol and laparoscopic surgery.

To further reduce the surgical trauma, SILS has emerged as a tool to minimize the numbers of scars and provide significantly better cosmetic results with no significant differences compared to CLS with regard to other shortterm outcomes $(13,32,33)$. However, several obstacles to the generalization of this new technique still exist, such as conflicts of surgical devices, loss of triangulation, and in-line viewing. Thus, SILS plus one was introduced as a bridge to SILS. Adding an additional port may overcome the limitations of SILS, thereby shortening the learning curve while preserving the minimally invasive benefits of SILS $(34,35)$. In addition, the advantage of adding an extra port is particularly evident in anterior resection for rectal cancer. For example, using one additional port for the linear stapler could permit the surgeon to transect the proximal rectum easier, consequently reducing the number of linear staplers for anterior resection and the risk of anastomosis leakage (36-38).

Our center began to apply SILS plus one technique to elective patients with colorectal cancer since 2013 . Our previous RCT (35) showed that surgical outcomes, including length of the proximal and distal margins, and the number of lymph nodes harvested, were similar between the CLS and SILS plus one groups for patients with rectosigmoid cancer whereas SILS plus one group achieved shorter total operating time, less blood loss, and less pain. Similar findings were also reported by Song et al. (38) and Yu et al. (34). Besides, our previous retrospective studies have also concluded that SILS plus one is a better choice than CLS or SILS for rectosigmoid cancer because it can minimize surgical trauma and reduce technical difficulties at the same time (39).

Taking together, SILS plus one and ERAS care are both increasingly preferred in the treatment of colorectal cancer. Our team has accumulated rich experience in performing the SILS plus one technique and applying ERAS perioperative management. However, to the best of our knowledge, none of the current exiting literature has researched SILS plus one colorectal resection assisted with the ERAS protocol. Thus, this study will be the first to evaluate SILS plus one combined with ERAS protocol for colorectal cancer. In conclusion, we hypothesized that combining advantages of minimal invasiveness and enhanced recovery of SILS plus one assisted with ERAS protocol might serve patients with colorectal cancer as a more safe, economic, feasible, and rapid alternative surgery strategy.

\section{Trial status}

Trial recruitment was ongoing at the time of manuscript submission. This manuscript refers to the second version of the full study protocol issued on 15 February 2020. The 
trial will last from 15 February 2020 to 15 February 2022.

\section{Acknowledgments}

Funding: This work was supported by the grant from Natural Science Foundation of Guangdong Province of China (2018A030313537).

\section{Footnote}

Reporting Checklist: The authors have completed the SPIRIT reporting checklist. Available at https://dx.doi. org/10.21037/tcr-21-1361

Conflicts of Interest: All authors have completed the ICMJE uniform disclosure form (available at https://dx.doi. org/10.21037/tcr-21-1361). The authors have no conflicts of interest to declare.

Etbical Statement: The authors are accountable for all aspects of the work in ensuring that questions related to the accuracy or integrity of any part of the work are appropriately investigated and resolved. The study will be conducted in accordance with the Declaration of Helsinki (as revised in 2013). The study protocol was approved by Medical Ethics Committee of The Medical Ethics Committee of Nanfang Hospital (reference number: NFEC-2019-156). The Medical Ethics Committee of Nanfang Hospital will be informed of significant protocol amendments. Written informed consent will be obtained from all individual participants for publication of this study.

Open Access Statement: This is an Open Access article distributed in accordance with the Creative Commons Attribution-NonCommercial-NoDerivs 4.0 International License (CC BY-NC-ND 4.0), which permits the noncommercial replication and distribution of the article with the strict proviso that no changes or edits are made and the original work is properly cited (including links to both the formal publication through the relevant DOI and the license). See: https://creativecommons.org/licenses/by-nc-nd/4.0/.

\section{References}

1. Dekker E, Tanis PJ, Vleugels JLA, et al. Colorectal cancer. Lancet 2019;394:1467-80.

2. Wilmore DW, Kehlet H. Management of patients in fast track surgery. BMJ 2001;322:473-6.
3. Li Q, Du L, Lu L, et al. Clinical Application of Enhanced Recovery After Surgery in Perioperative Period of Laparoscopic Colorectal Cancer Surgery. J Laparoendosc Adv Surg Tech A 2019;29:178-83.

4. Ren L, Zhu D, Wei Y, et al. Enhanced Recovery After Surgery (ERAS) program attenuates stress and accelerates recovery in patients after radical resection for colorectal cancer: a prospective randomized controlled trial. World J Surg 2012;36:407-14.

5. Wang J, Luo Y, Wang Q, et al. Evaluation of the application of laparoscopy in enhanced recovery after surgery (ERAS) for gastric cancer: a Chinese multicenter analysis. Ann Transl Med 2020;8:543.

6. Feng F, Li XH, Shi H, et al. Fast-track surgery combined with laparoscopy could improve postoperative recovery of low-risk rectal cancer patients: a randomized controlled clinical trial. J Dig Dis 2014;15:306-13.

7. Munk-Madsen P, Eriksen JR, Kehlet H, et al. Why still in hospital after laparoscopic colorectal surgery within an enhanced recovery programme? Colorectal Dis 2019;21:1438-44.

8. Zhao JH, Sun JX, Gao P, et al. Fast-track surgery versus traditional perioperative care in laparoscopic colorectal cancer surgery: a meta-analysis. BMC Cancer 2014;14:607.

9. Gustafsson UO, Scott MJ, Schwenk W, et al. Guidelines for perioperative care in elective colonic surgery: Enhanced Recovery After Surgery (ERAS(®)) Society recommendations. World J Surg 2013;37:259-84.

10. Shida D, Tagawa K, Inada K, et al. Enhanced recovery after surgery (ERAS) protocols for colorectal cancer in Japan. BMC Surg 2015;15:90.

11. Veldkamp R, Kuhry E, Hop WC, et al. Laparoscopic surgery versus open surgery for colon cancer: shortterm outcomes of a randomised trial. Lancet Oncol 2005;6:477-84.

12. Lacy AM, García-Valdecasas JC, Delgado S, et al. Laparoscopy-assisted colectomy versus open colectomy for treatment of non-metastatic colon cancer: a randomised trial. Lancet 2002;359:2224-9.

13. Watanabe J, Ota M, Fujii S, et al. Randomized clinical trial of single-incision versus multiport laparoscopic colectomy. Br J Surg 2016;103:1276-81.

14. Poon JT, Cheung CW, Fan JK, et al. Single-incision versus conventional laparoscopic colectomy for colonic neoplasm: a randomized, controlled trial. Surg Endosc 2012;26:2729-34.

15. Kang BM, Park SJ, Lee KY, et al. Single-Port Laparoscopic Surgery Can Be Performed Safely and 
Appropriately for Colon Cancer: Short-Term Results of a Pilot Randomized Controlled Trial. J Laparoendosc Adv Surg Tech A 2017;27:501-9.

16. Huscher CG, Mingoli A, Sgarzini G, et al. Standard laparoscopic versus single-incision laparoscopic colectomy for cancer: early results of a randomized prospective study. Am J Surg 2012;204:115-20.

17. Edge SB, Compton CC. The American Joint Committee on Cancer: the 7th edition of the AJCC cancer staging manual and the future of TNM. Ann Surg Oncol 2010;17:1471-4.

18. Wang Y, Liu R, Zhang Z, et al. A safety study of transumbilical single incision versus conventional laparoscopic surgery for colorectal cancer: study protocol for a randomized controlled trial. Trials 2015;16:539.

19. ATS Committee on Proficiency Standards for Clinical Pulmonary Function Laboratories. ATS statement: guidelines for the six-minute walk test. Am J Respir Crit Care Med 2002;166:111-7.

20. Dindo D, Demartines N, Clavien PA. Classification of surgical complications: a new proposal with evaluation in a cohort of 6336 patients and results of a survey. Ann Surg 2004;240:205-13.

21. National Cancer Institute. Common Terminology Criteria for Adverse Events (CTCAE). Available online: https://ctep.cancer.gov/protocoldevelopment/electronic_ applications/ctc.htm\#ctc_50

22. Strasberg SM, Linehan DC, Hawkins WG. The accordion severity grading system of surgical complications. Ann Surg 2009;250:177-86.

23. Varadhan KK, Neal KR, Dejong CH, et al. The enhanced recovery after surgery (ERAS) pathway for patients undergoing major elective open colorectal surgery: a meta-analysis of randomized controlled trials. Clin Nutr 2010;29:434-40.

24. Jayne DG, Guillou PJ, Thorpe H, et al. Randomized trial of laparoscopic-assisted resection of colorectal carcinoma: 3-year results of the UK MRC CLASICC Trial Group. J Clin Oncol 2007;25:3061-8.

25. Colon Cancer Laparoscopic or Open Resection Study Group; Buunen M, Veldkamp R, et al. Survival after laparoscopic surgery versus open surgery for colon cancer: long-term outcome of a randomised clinical trial. Lancet Oncol 2009; 10:44-52.

26. Arezzo A, Passera R, Scozzari G, et al. Laparoscopy for rectal cancer reduces short-term mortality and morbidity: results of a systematic review and meta-analysis. Surg Endosc 2013;27:1485-502.
27. Theophilus M, Platell C, Spilsbury K. Long-term survival following laparoscopic and open colectomy for colon cancer: a meta-analysis of randomized controlled trials. Colorectal Dis 2014;16:O75-81.

28. Pędziwiatr M, Małczak P, Mizera M, et al. There is no difference in outcome between laparoscopic and open surgery for rectal cancer: a systematic review and metaanalysis on short- and long-term oncologic outcomes. Tech Coloproctol 2017;21:595-604.

29. Li J, Kong XX, Zhou JJ, et al. Fast-track multidisciplinary treatment versus conventional treatment for colorectal cancer: a multicenter, open-label randomized controlled study. BMC Cancer 2019;19:988.

30. Xu D, Li J, Song Y, et al. Laparoscopic surgery contributes more to nutritional and immunologic recovery than fast-track care in colorectal cancer. World J Surg Oncol 2015;13:18.

31. Veenhof AA, Vlug MS, van der Pas MH, et al. Surgical stress response and postoperative immune function after laparoscopy or open surgery with fast track or standard perioperative care: a randomized trial. Ann Surg 2012;255:216-21.

32. Kang BM, Kim HJ, Kye BH, et al. Multicenter, randomized single-port versus multiport laparoscopic surgery (SIMPLE) trial in colon cancer: an interim analysis. Surg Endosc 2018;32:1540-9.

33. Maggiori L, Tuech JJ, Cotte E, et al. Single-incision Laparoscopy Versus Multiport Laparoscopy for Colonic Surgery: A Multicenter, Double-blinded, Randomized Controlled Trial. Ann Surg 2018;268:740-6.

34. Yu H, Shin JY. Short-term outcomes following reducedport, single-port, and multi-port laparoscopic surgery for colon cancer: tailored laparoscopic approaches based on tumor size and nodal status. Int J Colorectal Dis 2016;31:115-22.

35. Wang Y, Deng H, Mou T, et al. Short-term outcomes of single-incision plus one-port laparoscopic versus conventional laparoscopic surgery for rectosigmoid cancer: a randomized controlled trial. Surg Endosc 2019;33:840-8.

36. Lim SW, Kim HJ, Kim CH, et al. Umbilical incision laparoscopic colectomy with one additional port for colorectal cancer. Tech Coloproctol 2013;17:193-9.

37. Kawamata F, Homma S, Minagawa N, et al. Comparison of single-incision plus one additional port laparoscopyassisted anterior resection with conventional laparoscopyassisted anterior resection for rectal cancer. World J Surg 2014;38:2716-23.

38. Song JM, Kim JH, Lee YS, et al. Reduced port 
laparoscopic surgery for colon cancer is safe and feasible in terms of short-term outcomes: comparative study with conventional multiport laparoscopic surgery. Ann Surg Treat Res 2016;91:195-201.

39. Liu R, Wang Y, Zhang Z, et al. Assessment of treatment

Cite this article as: Zhang X, Li G, Li X, Liang Z, Lan X, Mou T, Xu Z, Fu J, Wu M, Li G, Wang Y. Effect of single-incision plus one port laparoscopic surgery assisted with enhanced recovery after surgery on colorectal cancer: study protocol for a single-arm trial. Transl Cancer Res 2021;10(12):5443-5453. doi: $10.21037 /$ tcr-21-1361 options for rectosigmoid cancer: single-incision plus one port laparoscopic surgery, single-incision laparoscopic surgery, and conventional laparoscopic surgery. Surg Endosc 2017;31:2437-50. 